\title{
Design and simulation analysis of an improved wearable power knee exoskeleton
}

\author{
Fangzheng Wang1, Lei Yan', Jiang Xiao ${ }^{3}$, Lei Fan ${ }^{4}$ \\ School of Technology, Beijing Forestry University, Beijing, China \\ ${ }^{2}$ Corresponding author

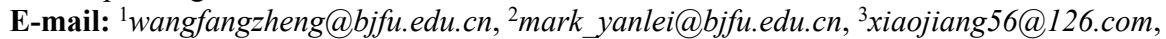 \\ ${ }^{4}$ fanlei@bjfu.edu.cn
}

Received 23 April 2018; received in revised form 11 April 2019; accepted 24 April 2019 DOI https://doi.org/10.21595/jve.2019.19920

Check for updates

Copyright $(C) 2019$ Fangzheng Wang, et al. This is an open access article distributed under the Creative Commons Attribution License, which permits unrestricted use, distribution, and reproduction in any medium, provided the original work is properly cited.

\begin{abstract}
The wearable lower limb power robotic exoskeleton is a device that can improve the human walking ability. In this paper, an improved exoskeleton device for knee joint is designed, including the improvement of mechanical structure and hydraulic cylinder. In order to verify the effectiveness of the improvement of the hydraulic cylinder, we have carried out the following studies. Firstly, in terms of mechanical structure, length adjusting device is added to meet the needs of different people. At the same time, a limit device is added to the knee joint to improve the safety performance and comfort. Secondly, the dynamics of the model is carried out by Lagrange, and the exoskeleton model is established for ADAMS motion simulation. The force of ADAMS simulation, the calculated by Lagrange equation and the force of the first edition of hydraulic cylinder are compared, and the force selection of hydraulic cylinder is analyzed. By comparison with the first edition, the optimization rate of the improved hydraulic cylinder reaches $8 \%$. Finally, in order to verify the rationality of ADAMS simulation and the effectiveness of hydraulic cylinder improvement, the wear test is carried out, the average errors of leg centroid in normal walking, wearing exoskeleton walking and ADAMS simulation data are compared. The average error rate is less than $10 \%$. The results show that the simulation model design is reasonable, and the effectiveness of the hydraulic cylinder improvement is verified. The exoskeleton device designed can well follow the human motion. The simulation analysis of the exoskeleton provides important parameters for the manufacture and it also provides theoretical basis for the later control theory.
\end{abstract}

Keywords: knee joint exoskeleton, dynamics analysis, Adams simulation.

\section{Introduction}

The wearable exoskeleton device has been widely used in all aspects of daily life and industry. For example, in military applications, an exoskeleton is mainly used to improve the operational ability of soldiers and ease the carrying of weapons [1]. In industrial applications, the exoskeletons are mainly used to reduce the labor intensity of workers. In addition, in the medical field, an exoskeleton can assist patients who are unable to walk [2]. However, some important factors, such as lightness quality, wear comfort and control flexibility, need to be improved.

A brief introduction of a few commercial exoskeleton robot is presented below.

The AlterG Bionic Leg ${ }^{\mathrm{TM}}$ is a wearable mobile auxiliary device, which is powered by batteries [3]. It can help patients walk and automatically identify the patients' movement intention [4, 5]. At the same time, the device can also help in the rehabilitation of patients with spinal injuries to help them walk, so as to enhance and improve their sports posture and enhance their ability to exercise.

The C-Brace ${ }^{\circledR}$ Orthotronic Mobility System is a passive knee ankle foot orthosis robotic exoskeleton for running support [6]. It can be used to help paralyzed patients recover by stretching the knee joint. The hydraulic resistance control is provided by adjusting the hydraulic resistance of the standing stage and the swing stage to provide the walking aid to the patients, help wearers run or climb stairs to satisfy the patients' walking desire $[7,8]$. 
A robotic knee exoskeleton robot has been designed in our Laboratory for the assistance of the elderly. The first version of the lower limb exoskeleton was designed, as shown in Fig. 1. However, some shortcomings in the trial restricted the cooperation ability of the robot with the patient, the structure was heavy, the operation was inconvenient, and the security was poor. Therefore, in this paper, the improved version is introduced. In order to verify the effectiveness of the hydraulic cylinder improvement, the dynamic analysis of the model is carried out by Lagrange, and the ADAMS motion simulation of the exoskeleton model is established. By comparison with the first edition, the optimization rate of the improved hydraulic cylinder reaches $8 \%$. In order to verify the rationality of ADAMS simulation and the effectiveness of hydraulic cylinder improvement, the wear test was carried out. The average errors of the center of mass of the legs in normal walking, wearing exoskeleton walking and ADAMS simulation data were compared, and the average error rate was less than $10 \%$. The results show that the simulation model design is reasonable and the effectiveness of the improvement of the hydraulic cylinder is verified.

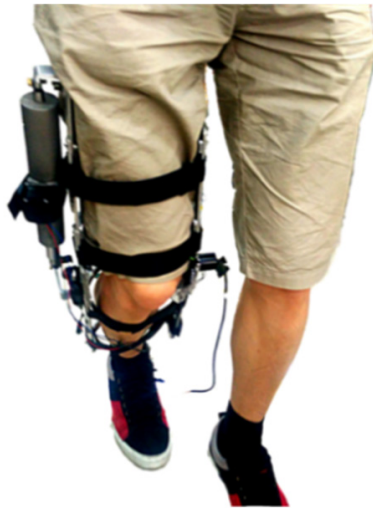

Fig. 1. The first version of the knee joint powered robotic exoskeleton

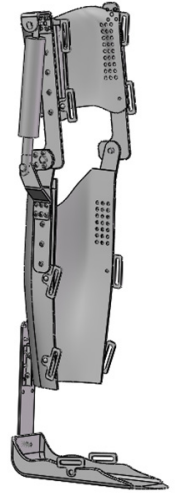

Fig. 2. The improved version of the robotic exoskeleton

\section{The structure design of lower limb exoskeleton}

The robotic exoskeleton is a device that combines a human with a machine. The improved version is diagrammatically depicted in Fig. 2, the knee joint of the lower extremities has one degree of freedom, and an actuator. The device was ergonomically designed for the comfort and convenience of the wearer. The structure of the whole device is optimized and improved to increase its quality. In addition, an adjustable device is used on the thighs and legs in order to meet the wear ability requirements of patients with different heights.

\subsection{The length adjusting of the device}

In order to wear more comfortably, the design of exoskeleton should be consistent with the size of human lower limbs, and the length of exoskeleton device can be adjusted according to the requirements. Fig. 3 shows the proportion of lower limbs relative to the total height of the human body. Let $H$ be the total height of the human body, the total height of the thigh relative to the human body is $0.245 \mathrm{H}$, and the length of the leg relative to the human body is $0.246 \mathrm{H}$ [9]. As shown in Fig. 3, the red line represents the position of the lower extremity exoskeleton device in the human body. The total length of the designed thigh relative to the human body is $0.12 \mathrm{H}$, and the length of the lower leg relative to the human body is $0.246 \mathrm{H}$. According to the height range from $160 \mathrm{~cm}$ to $180 \mathrm{~cm}$, for example. As shown in Table 1, the adjusting lengths of the thighs and legs of the exoskeleton device are calculated when $H=160 \mathrm{~cm}$ and $H=180 \mathrm{~cm} \mathrm{[10]}$.

According to Table 1, the structural parameters of the rod of exoskeleton device are determined:

- Adjustment range of thigh: $190 \mathrm{~mm}-220 \mathrm{~mm}$, 
- Adjustment range of calf: $390 \mathrm{~mm}-450 \mathrm{~mm}$.

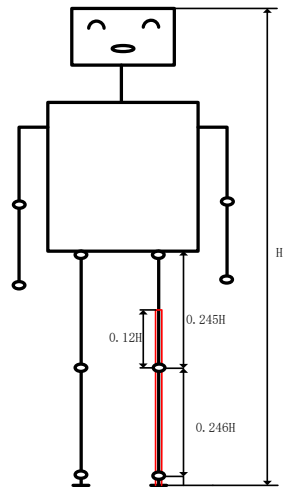

Fig. 3. The body with the height of the proportion

Table 1. Adjustment range of connecting rod of exoskeleton robot

\begin{tabular}{|c|c|c|c|c|}
\hline Connecting rod & $H$ & $1600 \mathrm{~mm}$ & $1800 \mathrm{~mm}$ & Range \\
\hline Length of thigh & $0.12 \mathrm{H}$ & $190 \mathrm{~mm}$ & $220 \mathrm{~mm}$ & $190 \mathrm{~mm}-220 \mathrm{~mm}$ \\
\hline Length of the calf & $0.246 \mathrm{H}$ & $390 \mathrm{~mm}$ & $450 \mathrm{~mm}$ & $390 \mathrm{~mm}-450 \mathrm{~mm}$ \\
\hline
\end{tabular}

Four adjusting holes are designed by adjusting device. The adjusting interval of thigh is $10 \mathrm{~mm}$, so the length of thigh can be fixed to $190 \mathrm{~mm}, 200 \mathrm{~mm}, 210 \mathrm{~mm}$ and $220 \mathrm{~mm}$. The adjusting interval of calf is $20 \mathrm{~mm}$, so the length of the calf can be fixed to $390 \mathrm{~mm}, 410 \mathrm{~mm}$, $430 \mathrm{~mm}$ and $450 \mathrm{~mm}$. The position of the adjusting hole is shown in Fig. 4.

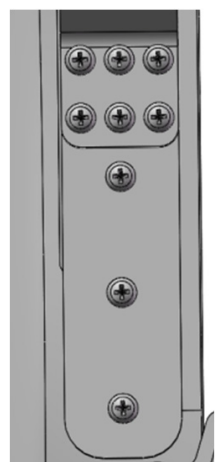

a)

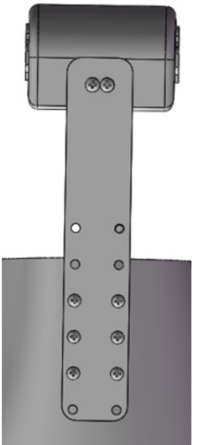

b)

Fig. 4. a) The adjusting device of the length of the thigh, b) the adjusting device of the length of the calf

\subsection{The limiting device of the knee}

When designing the exoskeleton, careful consideration should be given to safety issues. Accordingly, a knee joint rotation limiting device is necessary to include in the design. In the direction of sagittal movement, the rotation range of the knee joint is between $0-80^{\circ}[3,11]$. In order to avoid the knee joint going beyond this range, we designed a groove in the knee joint rotation device, so the exoskeleton can only move within this range, as shown in Fig. 5(a). Its working principle is shown in Fig. 5(b). When the knee joint rotates, points A and B rotate around the center point $\mathrm{O}$ in the groove mechanism respectively. When $\mathrm{A}$ rotates to $\mathrm{C}$ and $\mathrm{B}$ rotates to $\mathrm{D}$, they reach the ultimate rotation position, which helps to realize the limitation of the range of motion of the joint, so as to play a role in human safety protection. 


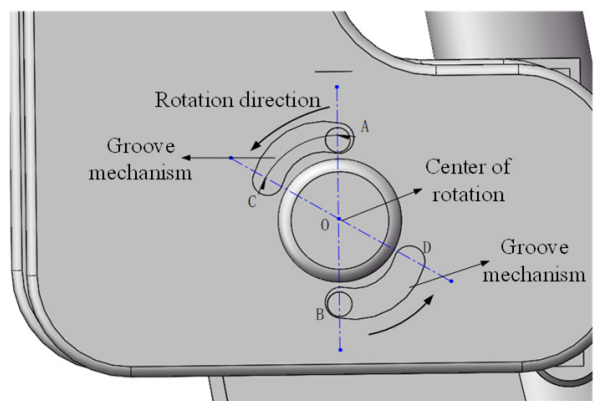

a) Working sketch

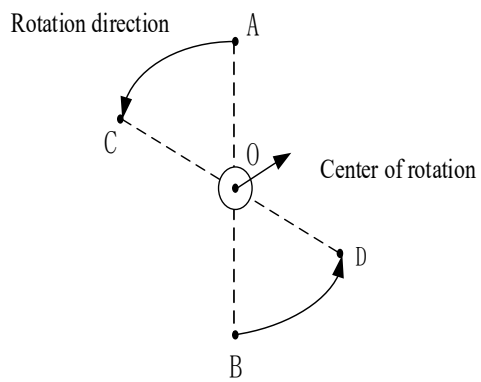

b) Working principle diagram

Fig. 5. Knee joint rotation limiting device

\subsection{The driving mechanism}

The exoskeleton device not only needs to be small in size and have a compact structure, but also must be lightweight. Therefore, the actuator uses an electric hydraulic cylinder, as shown in Fig. 6. First, the hydraulic actuator is a linear motion actuator, which greatly simplifies the design of the mechanical structure, and can transform mechanical energy into human motion and provide assistance. Second, it is small in size and smooth motion, making the whole structure more compact and natural $[12,13]$. The rod parts of the hydraulic actuating structure were theoretically analyzed. Through calculation, the normal rated thrust of the hydraulic cylinder is is $600 \mathrm{~N}$.

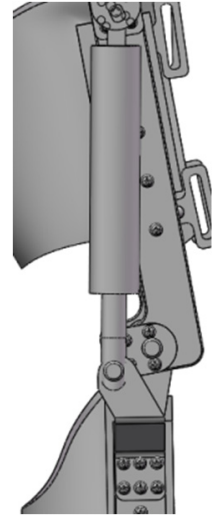

a)

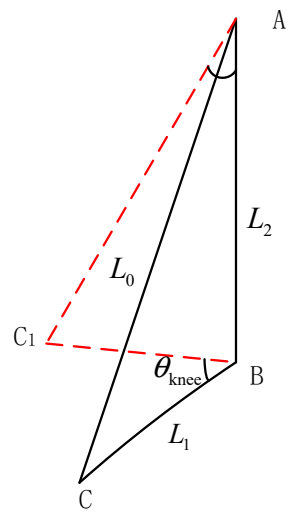

b)

Fig. 6. Knee driving linear motion actuator with hydraulic cylinder and hydraulic cylinder motion diagram

As shown in Fig. 6, to establish the relationship between the hydraulic cylinder and the installation location:

$\theta=\angle A B C-\theta_{\text {knee }}$

$\theta$ Comprises two straight lines $L_{1}$ and $L_{2}$ angle type, among them, the $L_{1}$ length is $38 \mathrm{~mm}$ and $L_{2}$ length is $189 \mathrm{~mm}$. $\theta_{\text {knee }}$ is the knee joint angle

In the case of the cosine law $\triangle A B C$, the length of the driver can be obtained:

$L_{0}=\sqrt{L_{1}^{2}+L_{2}^{2}-2 L_{1} L_{2} \cos \theta}$

However, the length of the drive must meet the Eq. (3) below: 
$L_{\min } \leq L_{0} \leq\left(L_{\min }+d\right)$,

when the knee joint hydraulic cylinder is at the two limit positions, the journey is the largest. According to human kinematics parameters, the rotation range of human knee joint is $0-80^{\circ}$ [14], with 0 and 80 generation into the type (2). The length of the hydraulic cylinder change range was calculated to be from $195 \mathrm{~mm}-220 \mathrm{~mm}$, with the largest travel distance being $25 \mathrm{~mm}$. The maximum driving torque of the walking aid mechanism should be the same as that of the driving body. The average weight of the normal human body is $60 \mathrm{~kg}[7,15]$. The proportion of the human body lower limbs relative to the whole human body is $38.68 \%$ [16]:

$T_{1}=\mathrm{m} \times 38.68 \% \times t \times 1000=60 \times 38.68 \% \times 0.98 \times 1000=22743.84 \mathrm{~N} . \mathrm{mm}$.

Among them, $t$ is the unit joint moment, the value is $0.98 \mathrm{~N} / \mathrm{kg}$. Finding the Maximum Driving Torque $T_{1}$, According to the Eq. (5):

$T_{1}=F_{1} \cdot L_{1}$

Given the lengths of the maximum driving torques $T_{1}$ and $L_{1}$, the maximum pulling force at the knee joint can be calculated. as follows the Eq. (6):

$F_{1}=\frac{T_{1}}{L_{1}}=\frac{22743.84}{38}=598.52 \mathrm{~N}$.

Accordingly, the selected driving force of the hydraulic cylinder is $600 \mathrm{~N}$.

\section{Lower limb exoskeleton dynamics analysis and simulation}

In order to verify the practicality of the design of the hydraulic cylinders and the mechanisms, the Lagrange method is adopted, and a simulation is conducted using the ADAMS software.

\subsection{Dynamics analysis}

First, in the process of dynamic analysis, we use Lagrange multipliers for dynamic analysis based on Fig. 9. The Lagrange equation is as shown in Eq. (7) and Eq. (8):

$$
\begin{aligned}
\tau_{i} & =\frac{d}{d_{t}}\left(\frac{\partial L}{\partial \dot{q}_{i}}\right)-\frac{\partial L}{\partial q_{i}}, \quad(i=1,2, \ldots, n), \\
L & =E_{K}-E_{P} .
\end{aligned}
$$

Therefore, the formulas to calculate the kinetic energy and the potential energy are as shown below.

Thigh connecting rod is as shown in Eq. (9) and Eq. (10):

$$
\begin{aligned}
& E_{k 1}=\frac{1}{2}\left(m_{1}+M_{1}\right) l_{c 1}^{2} \dot{\theta}_{1}^{2}-\frac{1}{2} m_{a} l_{c 1}^{2} \dot{\theta}_{1}^{2}, \\
& E_{P 1}=\left(m_{1}+M_{1}\right) g l_{c 1} \cos \theta_{1}-m_{a} g l_{1} \cos \theta_{1} .
\end{aligned}
$$

The crus connecting rod is as shown in Eq. (11) and Eq. (12):

$$
\begin{aligned}
& E_{k 2}=\frac{1}{2}\left(m_{2}+M_{2}\right)\left[l_{c 1}^{2} \dot{\theta}_{1}^{2}+l_{c 2}^{2}\left(\dot{\theta}_{1}+\dot{\theta}_{2}\right)^{2}-2 l_{1} l_{c 1}\left(\dot{\theta}_{1}+\dot{\theta}_{2}\right) \dot{\theta}_{1} \cos \dot{\theta}_{2}\right] \\
& E_{P 2}=\left(m_{2}+M_{2}\right) g\left[-l_{1} \cos \theta_{1}+l_{c 2} \cos \left(\theta_{1}+\theta_{2}\right)\right] .
\end{aligned}
$$


The expression of the Lagrange function can be obtained from the above formulas, as shown in Eq. (13):

$L=E_{K 1}+E_{K 2}-\left(E_{P 1}+E_{P 2}\right)$.

In the calculation, we can assume that the ankle as a passive joint, which rotates with the rotation of the knee joint. Accordingly, we can consider the following part of the knee joint as a whole connecting rod, and the unilateral robotic exoskeleton model can be simplified as a two-link model, as follows Eq. (14) and Eq. (15):

$$
\begin{aligned}
& E_{K 2}=\frac{1}{2}\left(m_{2}+M_{2}\right)\left[l_{c 1}^{2} \dot{\theta}_{1}^{2}+l_{c 2}^{2}\left(\dot{\theta}_{1}+\dot{\theta}_{2}\right)^{2}-2 l_{1} l_{c 1}\left(\dot{\theta}_{1}+\dot{\theta}_{2}\right) \dot{\theta}_{1} \cos \dot{\theta}_{2}\right] \\
& \quad+\frac{1}{2} m_{f}\left[l_{c 1}^{2} \dot{\theta}_{1}^{2}+l_{c 2}^{2}\left(\dot{\theta}_{1}+\dot{\theta}_{2}\right)^{2}-2 l_{1} l_{c 1}\left(\dot{\theta}_{1}+\dot{\theta}_{2}\right) \dot{\theta}_{1} \cos \dot{\theta}_{2}\right], \\
& E_{P 2}=\left(m_{2}+M_{2}\right) g\left[-l_{1} \cos \theta_{1}+l_{c 2} \cos \left(\theta_{1}+\theta_{2}\right)\right]+m_{f} \mathrm{~g}\left[-l_{1} \cos \theta_{1}+l_{c 2} \cos \left(\theta_{1}+\theta_{2}\right)\right] .
\end{aligned}
$$

The Lagrange function of the single side two-link system of the mechanism $L$ is as shown in Eq. (16):

$$
\begin{aligned}
L & =E_{K 1}+E_{K 2}-\left(E_{P 1}+E_{P 2}\right)=\frac{1}{2}\left(m_{1}+M_{1}\right) l_{c 1}^{2} \dot{\theta}_{1}^{2}-\frac{1}{2} m_{a} l_{c 2}^{2} \dot{\theta}_{1}^{2} \\
& +\frac{1}{2}\left(m_{1}+M_{1}\right)\left[l_{c 1}^{2} \dot{\theta}_{1}^{2}+l_{c 1}^{2}\left(\dot{\theta}_{2}+\dot{\theta}_{1}\right)^{2}-2 l_{1} l_{c 1}\left(\dot{\theta}_{2}+\dot{\theta}_{1}\right) \dot{\theta}_{1} \cos \dot{\theta}_{2}\right] \\
& +\frac{1}{2} m_{f}\left[l_{c 1}^{2} \dot{\theta}_{1}^{2}+l_{c 2}^{2}\left(\dot{\theta}_{2}+\dot{\theta}_{1}\right)^{2}-2 l_{1} l_{c 1}\left(\dot{\theta}_{2}+\dot{\theta}_{1}\right) \dot{\theta}_{1} \cos \dot{\theta}_{2}\right]+\left(m_{1}+M_{1}\right) g l_{c 1} \cos \theta_{1} \\
& -m_{a} g l_{1} \cos \theta_{1}-\left(m_{2}+M_{2}\right) g\left[-l_{1} \cos \theta_{1}+l_{c 2} \cos \left(\theta_{2}+\theta_{1}\right)\right] \\
& -m_{f} g\left[-l_{1} \cos \theta_{1}+l_{c 2} \cos \left(\theta_{2}+\theta_{1}\right)\right] .
\end{aligned}
$$

According to the above formula, we need to take the derivative respectively:

$$
\frac{\partial L}{\partial \theta_{1}}, \frac{\partial L}{\partial \theta_{2}}, \frac{\partial L}{\partial \dot{\theta}_{1}}, \frac{\partial L}{\partial \dot{\theta}_{2}}, \frac{d}{d_{t}} \frac{\partial L}{\partial \dot{\theta}_{1}}, \frac{d}{d_{t}} \frac{\partial L}{\partial \dot{\theta}_{2}} .
$$

The dynamic expression of the torque of the hip joint $T_{1}$ and the torque of the knee joint $T_{2}$ is as shown in Eq. (17) and Eq. (18):

$T_{1}=D_{11} \ddot{\theta}_{1}+D_{12} \ddot{\theta}_{2}+D_{111} \dot{\theta}_{1}+D_{122} \dot{\theta}_{2}+D_{112} \dot{\theta}_{1} \dot{\theta}_{2}+D_{121} \dot{\theta}_{2} \dot{\theta}_{1}+D_{1}$

$T_{2}=D_{21} \ddot{\theta}_{1}+D_{22} \ddot{\theta}_{2}+D_{211} \dot{\theta}_{1}+D_{222} \dot{\theta}_{2}+D_{212} \dot{\theta}_{1} \dot{\theta}_{2}+D_{221} \dot{\theta}_{2} \dot{\theta}_{1}+D_{2}$.

The equations of $T_{1}$ and $T_{2}$ expressed in matrix form are as shown in Eq. (19):

$$
\left[\begin{array}{l}
T_{1} \\
T_{2}
\end{array}\right]=\left[\begin{array}{ll}
D_{11} & D_{12} \\
D_{21} & D_{22}
\end{array}\right]\left[\begin{array}{l}
\ddot{\theta}_{1} \\
\ddot{\theta}_{2}
\end{array}\right]+\left[\begin{array}{ll}
D_{111} & D_{122} \\
D_{211} & D_{222}
\end{array}\right]\left[\begin{array}{l}
\dot{\theta}_{1} \\
\dot{\theta}_{2}
\end{array}\right]+\left[\begin{array}{ll}
D_{112} & D_{121} \\
D_{212} & D_{221}
\end{array}\right]\left[\begin{array}{l}
\dot{\theta}_{1} \dot{\theta}_{2} \\
\dot{\theta}_{2} \dot{\theta}_{1}
\end{array}\right]+\left[\begin{array}{c}
D_{1} \\
D_{2}
\end{array}\right]
$$

Through the above calculations, we provide a reliable theoretical basis for the motion state of the single lower extremity robotic exoskeleton, and provide a reference for the selection of hydraulic cylinders. In addition, the calculations also provide a theoretical basis and reference for the development and design of the control system.

\subsection{Adams simulation analysis}

For the purposes of the simulation, the three-dimensional model fabricated using SolidWorks 
is imported into the ADAMS software [17, 18]. The properties need to be edited, so the length and the center of gravity of each component in the ADAMS software, and the parameters are set up as shown in Table 2.

Table 2. Normal human body size and quality

\begin{tabular}{|c|c|c|}
\hline The section name of the body & Mass or centroid (mean value) & Length \\
\hline \multirow{2}{*}{ Calf } & $2.20 \mathrm{~kg}$ & \multirow{2}{*}{$344 \mathrm{~mm}$} \\
\cline { 2 - 2 } & $224.1 \mathrm{~mm}$ & \\
\cline { 2 - 3 } Thigh & $8.50 \mathrm{~kg}$ & \multirow{2}{*}{$438 \mathrm{~mm}$} \\
\hline Torso & $254.5 \mathrm{~mm}$ & $466.8 \mathrm{~mm}$ \\
\hline Upper arm & $26.37 \mathrm{~kg}$ & $284 \mathrm{~mm}$ \\
\hline Fore arm & $1.46 \mathrm{~kg}$ & $213 \mathrm{~mm}$ \\
\hline
\end{tabular}

In the simulation process, we need to limit the rotation of the upper part of the human body to maintain balance. The friction coefficient is 0.6 in the human body and the ground $[19,20]$. The driving data of each joint is set according to the standard drive function. The model is depicted in Fig. 7.

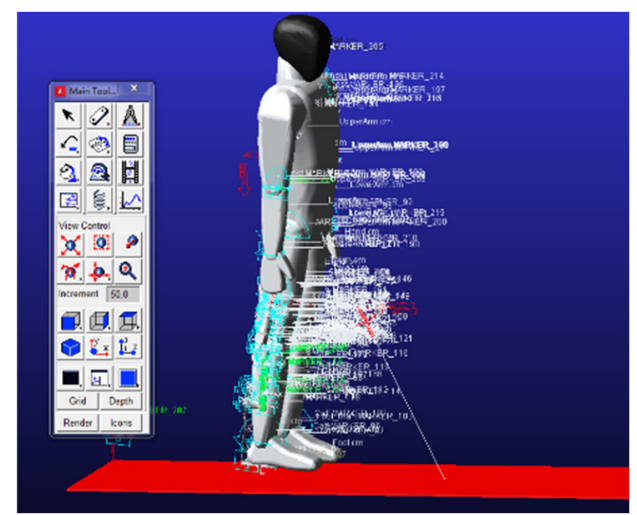

Fig. 7. Wearable exoskeleton model and the human body

After the simulation, the data processing is performed by the ADAMS/postprocessor. As shown in Fig. 8, it is the theoretical angle of knee exoskeleton rotation and the angle data of ADAMS simulation. From the analysis of simulation data, it is found that the rotation angle of human knee joint is between 0 and 80 degrees, and it changes periodically. Through comparison, the error between simulation data and theoretical angle is within 0-6 degrees range, which shows that the simulation data of human joint angle accords with human kinematics. In addition, through the simulation data, it can be seen that the design of the knee joint limitation device meets the actual needs.

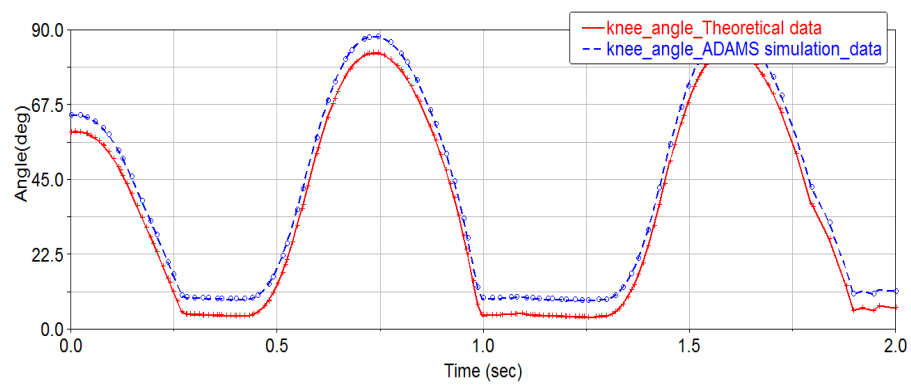

Fig. 8. Knee joint angle and the rotation angle of the Adams simulation 
As shown in Fig. 9, three force curves are compared, including the hydraulic cylinder driving force simulated by ADAMS, the hydraulic cylinder driving force of the first edition and the Lagrange dynamic theory analysis. In Fig. 9, it can be concluded that the driving force trends of the three cylinders are similar and have good consistency. For the driving force of knee joint, the calculated moment of Lagrange equation is in good agreement with the driving force obtained by ADAMS simulation, which proves the correctness of the theoretical analysis of Lagrange dynamics. The maximum driving force of the hydraulic cylinder simulated by ADAMS is $560 \mathrm{~N}$, and the maximum driving force of the hydraulic cylinder in the first edition is $650 \mathrm{~N}$. Using ADAMS simulation data as standard, the average error of Lagrange is $10 \mathrm{~N}$, the average error of driving force of the first edition is $50 \mathrm{~N}$, and the optimal driving force of the selected hydraulic cylinder is $8 \%$.

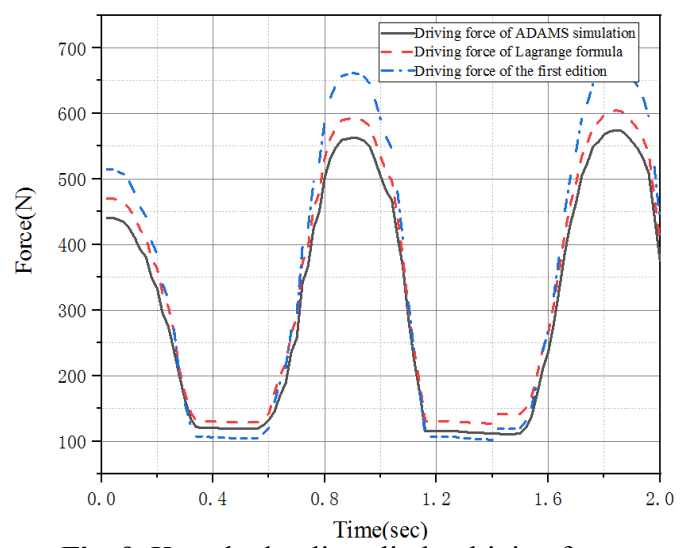

Fig. 9. Knee hydraulic cylinder driving force

\section{Results verification}

In order to verify the rationality of ADAMS simulation and the effectiveness of hydraulic cylinder improvement. The wear comparison experiment is needed. The influence of exoskeleton on human walking gait is mainly a comparative analysis of gait data with periodic variation. In this experiment, we used the data of human normal walking, wearing exoskeleton walking and ADAMS simulation to analyze the displacement curves in the $Y$ and $Z$ axis of the sagittal knee joint. As shown in Fig. 10, it is the human walking test with wearing exoskeleton.

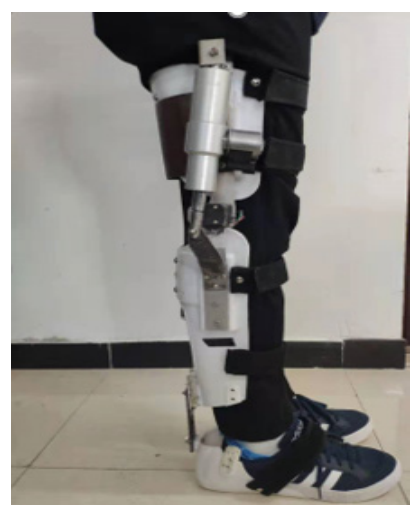

Fig. 10. The human walking test with wearing exoskeleton

As shown in Fig. 11, it is the change curve of center of mass displacement in $Y$ and $Z$ directions of normal walking, wearing exoskeleton walking and ADAMS simulation, and there is 
good consistency between them. The $Y$ direction represents the vertical displacement and the $Z$ direction represents the forward displacement.

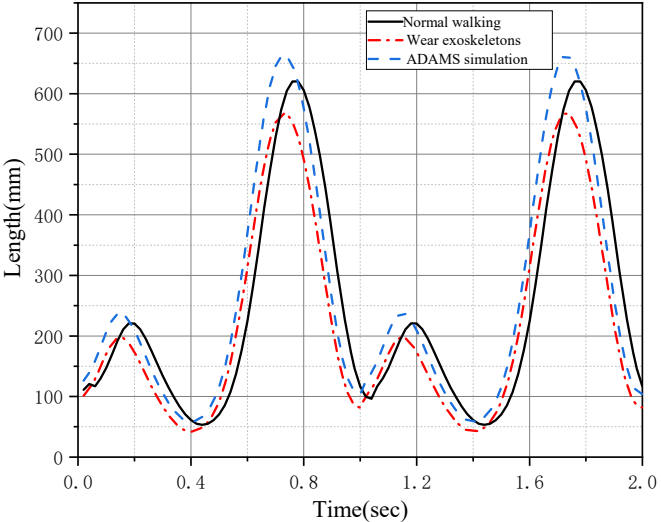

a) $Y$-axis centroid displacement curve

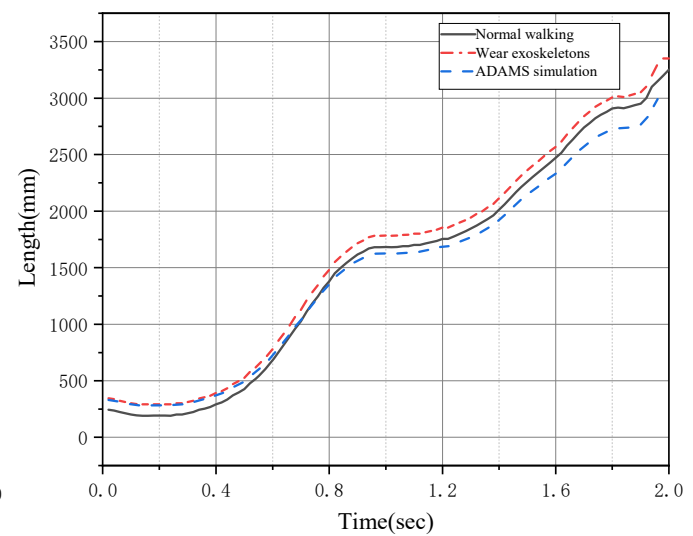

b) $Z$-axis centroid displacement curve

Fig. 11. Comparison of centroid changes of $Y$ and $Z$ axis

Using the normal walking data as the standard, as shown in Table 3, the average displacement errors of exoskeleton and normal walking in $Y$-axis is $10.5 \mathrm{~mm}$, the average displacement errors of exoskeleton and normal walking in $Z$-axis is $7.8 \mathrm{~mm}$. The average displacement errors of ADAMS simulation and normal walking in $Y$-axis is $9.3 \mathrm{~mm}$, the average displacement errors of ADAMS simulation and normal walking in $Z$-axis is $8.6 \mathrm{~mm}$. The average error is less than $10 \%$, the average error is within the required accuracy range. The results show that the ADAMS simulation model is reasonable, and the dynamic model can meet the requirements, which verifies the effectiveness of hydraulic cylinder improvement.

Table 3. Comparison of exoskeleton and ADAMS with normal walking

\begin{tabular}{|c|c|c|c|c|}
\hline \multirow{2}{*}{ Data } & \multicolumn{2}{|c|}{$\begin{array}{c}\text { The average displacement errors of } \\
\text { exoskeleton and normal walking }\end{array}$} & $\begin{array}{c}\text { The average displacement errors of ADAMS } \\
\text { simulation and normal walking }\end{array}$ \\
\cline { 2 - 5 } & $Y$-axis & $Z$-axis & $Y$-axis & $Z$-axis \\
\hline Maximum error & $15.5 \mathrm{~mm}$ & $18.5 \mathrm{~mm}$ & $19.8 \mathrm{~mm}$ & $17.5 \mathrm{~mm}$ \\
\hline Minimum error & $3.2 \mathrm{~mm}$ & $2.1 \mathrm{~mm}$ & $4.2 \mathrm{~mm}$ & $2.4 \mathrm{~mm}$ \\
\hline Average error & $10.5 \mathrm{~mm}$ & $7.8 \mathrm{~mm}$ & $9.3 \mathrm{~mm}$ & $8.6 \mathrm{~mm}$ \\
\hline
\end{tabular}

\section{Conclusions}

In this paper, an improved exoskeleton device for knee joint is designed, including the improvement of mechanical structure and hydraulic cylinder. In terms of mechanical structure, adjusting device and safety limit device are added. In order to verify the effectiveness of the hydraulic cylinder improvement, the dynamic analysis of the model is carried out by Lagrange, and the ADAMS simulation of the exoskeleton model is established. Three force curves were compared, including the force of ADAMS simulation, the calculated by Lagrange equation and the force of the first edition of hydraulic cylinder. Using the data simulated by ADAMS as the standard, the optimal driving force of the selected hydraulic cylinder was $8 \%$. In order to verify the rationality of ADAMS simulation and the effectiveness of hydraulic cylinder improvement, the wear test was carried out. The average error rate is less than $10 \%$. Therefore, the results show that the ADAMS simulation model is reasonable, and the dynamic model can meet the requirements, which verifies the effectiveness of hydraulic cylinder improvement. It also provides theoretical basis for the later control theory. 


\section{Acknowledgements}

This study is financially supported by the Fundamental Research Funds for the Central Universities (No. 2015ZCQ-GX-03).

\section{References}

[1] Vital J. P., et al. Combining discriminative spatiotemporal features for daily life activity recognition using wearable motion sensing suit. Pattern Analysis and Applications, Vol. 20, Issue 4, 2017, p. 1179-1194.

[2] Witte K. A., Fatschel A. M., Collins S. H. Design of a lightweight, tethered, torque-controlled knee exoskeleton. International Conference on Rehabilitation Robotics, London, 2017.

[3] Lu R., Li Z., Su C. Y., Xue A. Development and learning control of a human limb with a rehabilitation exoskeleton. IEEE Transactions on Industrial Electronics, Vol. 61, Issue 7, 2014, p. 3776-3785.

[4] Horst R. W. A bio-robotic leg orthosis for rehabilitation and mobility enhancement. Annual International Conference of the IEEE Engineering in Medicine and Biology Society, 2009.

[5] Xiao Y., Wang X., Li Z., Zhao Y. A method of kinematic analysis and singularity elimination for upper-limb exoskeleton. Jiqiren/Robot, Vol. 38, Issue 1, 2016, p. 33-40.

[6] Xu X., Hou L., Huang X., Zhang W. Design and research of a wearable robot for upper limbs rehabilitation based on exoskeleton. Jiqiren/Robot, Vol. 36, Issue 2, 2014, p. 147-155.

[7] Bai K. Design of a compliant knee-motion actuator for lower extremity exoskeletons. IEEE International Conference on Advanced Intelligent Mechatronics, Canada, 2016.

[8] Shamaei K., et al. Biomechanical effects of stiffness in parallel with the knee joint during walking. IEEE Transactions on Biomedical Engineering, Vol. 62, Issue 10, 2015, p. 2389-2401.

[9] Kirsch N. A., et al. Model-based dynamic control allocation in a hybrid neuroprosthesis. IEEE Transactions on Neural Systems and Rehabilitation Engineering, Vol. 26, Issue 1, 2018, p. 224-232.

[10] Yin Y. H., Fan Y. J., Xu L. D. Emg and Epp-integrated human-machine interface between the paralyzed and rehabilitation exoskeleton. IEEE Transactions on Information Technology in Biomedicine, Vol. 16, Issue 4, 2012, p. 542-549.

[11] Dollar A., Herr H. Lower extremity exoskeletons and active orthoses: challenges and state of the art. IEEE Transactions on Robotics, Vol. 24, Issue 1, 2008, p. 144-158.

[12] Martínez A., Lawson B., Goldfarb M. A Controller for guiding leg movement during overground walking with a lower limb exoskeleton. IEEE Transactions on Robotics, Vol. 34, Issue 1, 2018, p. 183-193.

[13] Lerner Z. F., Damiano D. L., And Bulea T. C. Relationship between assistive torque and knee biomechanics during exoskeleton walking in individuals with crouch gait. International Conference on Rehabilitation Robotics, London, 2017.

[14] Azocar A. F., Rouse E. J. Stiffness perception during active ankle and knee movement. IEEE Transactions on Biomedical Engineering, Vol. 64, Issue 12, 2017, p. 2949-2956.

[15] Viteckova S., Kutilek P., Jirina M. Wearable lower limb robotics: a review. Biocybernetics and Biomedical Engineering, Vol. 33, Issue 2, 2013, p. 96-105.

[16] Kim J. H., Han J. W., Kim D. Y., et al. Design of a walking assistance lower limb exoskeleton for paraplegic patients and hardware validation using CoP. International Journal of Advanced Robotic Systems, Vol. 10, Issue 9, 2013, p. 113-126.

[17] Hussain S., Xie S. Q., Jamwal P. K. Control of a robotic orthosis for gait rehabilitation. Robotics and Autonomous Systems, Vol. 61, Issue 9, 2013, p. 911-919.

[18] Anam K., Al-Jumaily A. Active exoskeleton control systems: state of the art. Procedia Engineering, Vol. 41, Issue 2, 2012, p. 988-994.

[19] Miao Y., Gao F., Pan D. State classification and motion description for the lower extremity exoskeleton SJTU-EX. Journal of Bionic Engineering, Vol. 11, Issue 2, 2014, p. 249-258.

[20] Shamaei K., Napolitano P. C., Dollar A. M. A quasi-passive compliant stance control Knee-AnkleFoot Orthosis. IEEE 13th International Conference on Rehabilitation Robotics, Washinggton, 2013. 


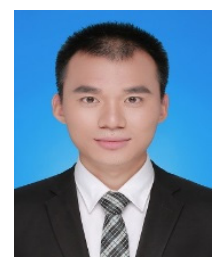

Fangzheng Wang received Master's degree in mechanical engineering from Beijing Forestry University, Beijing, China, in 2015. He is currently pursuing the Ph.D. degree at Beijing Forestry University. His current research interest is exoskeleton robot.

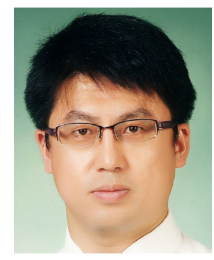

Lei Yan received his Ph.D. degree in electrical engineering and automation from Kyungpook National University, Daegu, Korea. He is an Associate Professor in School of Technology at Beijing Forestry University, Beijing, China. He has authored more than 60 papers published in various journals and completed 10 scientific research projects. His research interests focus on power-assisted exoskeleton robot, image recognition and artificial intelligence.

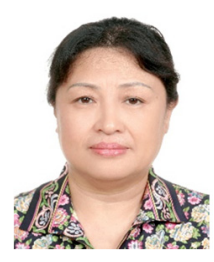

Jiang Xiao is currently a Professor with mechatronics engineering, School of Technology, Beijing Forestry University. She has completed several scientific research projects and published more than 30 papers in various journals. Her research interests focus on control of mechatronics system and wireless sensor network technology.

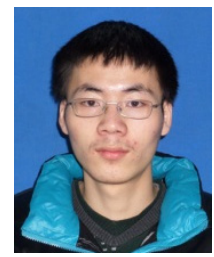

Lei Fan earned Bachelor's degree in mechatronics engineering from Northeast Forestry University, Harbin, China, in 2010. His current research interests include power assist exoskeleton system, automatic control system and embedded system. 